\title{
Changes in Known Statements After New Data is Added
}

\author{
Sylvia Encheva \\ Stord/Haugesund University College \\ Bjørnsonsg. 45, \\ 5528 Haugesund, \\ Norway
}

\begin{abstract}
Learning spaces are broadly defined as spaces with a noteworthy bearing on learning. They can be physical or virtual, as well as formal and informal. The formal ones are customary understood to be traditional classrooms or technologically enhanced active learning classrooms while the informal learning spaces can be libraries, lounges, cafés, etc.. Students' as well as lecturers' preferences to learning spaces along with the effects of these preferences on teaching and learning have been broadly discussed by many researchers. Yet, little is done to employ mathematical methods for drawing conclusions from available data as well as investigating changes in known statements after new data is added. To do this we suggest use of ordering rules and ordered sets theories.
\end{abstract}

Keywords-Ordering rules; Ordered sets; Implications

\section{INTRODUCTION}

Interest in building, teaching in, and researching the impact of technologically enhanced learning spaces appears to have grown exponentially, [3]. Learning spaces are usually divided into formal and informal, [7]. The first ones are customary understood to be classrooms while the latter can be libraries, lounges or cafés. At the same time, today's teaching and learning processes are heavily effected by an increasing use of laptop computers, smart phones and tablets. All these various opportunities can be viewed with respect to students' preferences and learning effectiveness. To do this we suggest support taken from ordering rules and ordered sets theories, [5].

Modern information technology tools allow good opportunities for collecting, storing and even classifying data. A number of scientific fields like for example automation and decision support systems, [4], require ordering of elements and rules.

The theory of ordering rules is often applied for ordering elements with respect to attributes' values. Ordering rules like "if the value of an object $x$ on an attribute a is ordered ahead of the value of another object $y$ on the same attribute, then $x$ is ordered ahead of $y "$, are presented in [10] and [11]. This rises a natural question on whether it is possible to combine several ordering rules and draw reasonable conclusions afterwards.

The rest of the paper goes as follows. Some terms from the fields of ordered sets and ordered relations are presented in Section II. Their application is discussed in Section III followed by a conclusion in Section IV.

\section{ORDERED SETS AND RELATIONS}

Two very interesting problems are considered in [2], namely the problem of determining a consensus from a group of orderings and the problem of making statistically significant statements about ordering.

A relation $I$ is an indifference relation when given $A I B$ neither $A>B$ nor $A<B$ has place in the componentwise ordering. A partial ordering whose indifference relation is transitive is called a weak ordering. A total ordering is a binary relation which is transitive, antisymmetric, and total $(p \leq q$ or $q \leq p)$.

If given two alternatives, a person is finally choosing only one. the natural extension of to more than two elements is known as the 'majority rule' or the 'Condorcet Principle'. A relation $R\left(L_{1}, L_{2}, \ldots, L_{k}\right)$ is constructed by saying that the pair $(a, b) \in R$ if $(a, b)$ belong to the majority of relations $L_{i}$.

The following three linear orderings

leading to

$$
\begin{array}{lll}
a & b & c \\
b & c & a \\
c & a & b
\end{array}
$$

$$
R=\{(a, b),(b, c),(c, a)\}
$$

(three-way tie), illustrate the 'paradox of voting'. A 'social welfare function' maps $k$-tuples of the set of linear orderings of any $b \subset A$ to single linear orderings of $B$, where $A$ is a set of at least three alternatives, [1].

Two elements $a$ and $b$ where $a \neq b$ and $a, b \in P$ are comparable if $a \leq b$ or $b \leq a$, and incomparable otherwise. If $\forall a, b$ where $a, b \in P$ are comparable, then $P$ is chain. If $\forall a, b$ where $a, b \in P$ are incomparable, then $P$ is antichain.

Interesting set-relational approach for computer administration of psychological investigations has been employed in [12].

Below we list some definitions and formulas as in [10]. The authors also introduce order relations into attribute values.

An information function $I_{a}$ is a total function mapping an object of $U$ to an exact value in $V_{a}$.

Definition 1: [9] An information table is a quadruple:

$$
I T=\left(U, A t,\left\{V_{a} \mid a \in A t\right\},\left\{I_{a} \mid a \in A t\right\}\right),
$$


where $U$ is a finite nonempty set of objects, $A t$ is a finite nonempty set of attributes, $V_{a}$ is a nonempty set of values for $a \in A t, I_{a}: U \rightarrow V_{a}$ is an information function.

Definition 2: Let $U$ be a nonempty set and $\succ$ be a binary relation on $U$. The relation $\succ$ is a weak order if it satisfies the two properties:

Asymmetry:

$$
x \succ y \Longrightarrow \neg(y \succ x),
$$

Negative transitivity:

$$
(\neg(x \succ y), \neg(y \succ z)) \Longrightarrow \neg(x \succ z) .
$$

An important implication of a weak order is that the following relation,

$$
x \sim y \Longleftrightarrow(\neg(x \succ y), \neg(y \succ x))
$$

is an equivalence relation. For two elements, if $x \sim y$, we say $x$ and $y$ are indiscernible by $\succ$. The equivalence relation $\sim$ induces a partition $U / \sim$ on $U$, and an order relation $\succ^{\star}$ on $U / \sim$ can be defined by

$$
[x]_{\sim} \succ^{\star}[y]_{\sim} \Longleftrightarrow x \succ y
$$

where $[x]_{\sim}$ is the equivalence class containing $x$. Any two distinct equivalence classes of $U / \sim$, can be compared since $\succ^{\star}$ is a linear order.

Definition 3: An ordered information table is a pair

$$
O I T=\left(I T,\left\{\succ_{a} \mid a \in A t\right\}\right),
$$

where $I T$ is a standard information table and $\succ_{a}$ is a weak order on $V_{a}$.

An ordering of values of a particular attribute a naturally induces an ordering of objects, namely, for $x, y \in U$ :

$$
x \succ_{\{a\}} y \Longleftrightarrow I_{a}(x) \succ_{a} I_{a}(y),
$$

where $\succ\{a\}$ denotes an order relation on $U$ induced by the attribute $a$. An object $x$ is ranked ahead of another object $y$ if and only if the value of $x$ on the attribute $a$ is ranked ahead of the value of $y$ on $a$. The relation $\succ_{\{a\}}$ has exactly the same properties as that of $\succ_{a}$. That is, $x$ is ranked ahead of $y$ if and only if $x$ is ranked ahead of $y$ according to all attributes in $A$.

Data mining in an ordered information table may be formulated as finding association between orderings induced by attributes.

Definition 4: Consider two subsets of attributes $A, B \subseteq$ At. For two expressions $\phi \in E(A)$ and $\psi \in E(B)$, an ordering rule is read "if $\phi$ then $\psi$ " and denoted by $\phi \Rightarrow \psi$. The expression $\phi$ is called the rule's antecedent, while the expression $\psi$ is called the rule's consequent.

\section{GRAPHICAL REPRESENTATION OF CORRELATIONS BETWEEN ORDERING RULES}

Recent enthusiasm for shifting the manner in which institutions of higher education approach and conceptualize classroom space has been fueled by a host of articles extolling the potential transformative power of formal learning spaces on teaching practices and learning outcomes, [3]. Handling such large amount of data resulting from both physical and mental processes requires solid well formalized techniques like f. ex. those included in the field of artificial intelligence.

It is worth mentioning that ordinary majority voting is unable to handle situations with incomplete or changing data. Ordered sets and ordering rules can accommodate both very nicely. They are particularly applicable in cases when some of the elements are compared while others are not or different conclusions are drawn by different groups and non of them should be ignored in a decision making process.

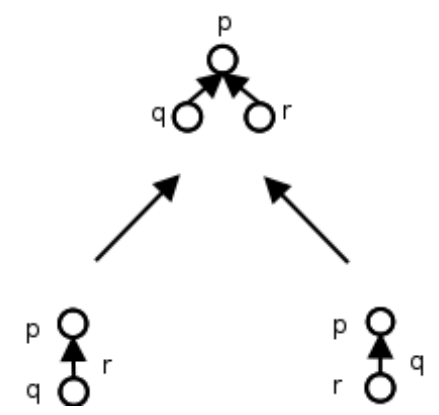

Fig. 1. $q$ and $r$ imply $p$

Suppose students are suggested to express their preferences with respect to two different learning spaces $x, y$ based on three attributes $a, b, c$. Their preferences related to learning spaces are first collected and conclusions can be drawn afterwards supported by ordered sets theory.

An ordering rule states how orderings of objects by attributes in $A$ determines orderings of objects by attributes in $B$. For example, an ordering rule,

$$
(a, \succ) \wedge(b, \preceq) \Rightarrow(c, \succ),
$$

can be re-expressed as

$$
x \succ_{\{a\}} y \wedge x \preceq_{\{b\}} y \Rightarrow x \succ_{\{c\}} y .
$$

That is, for two arbitrary objects $x$ and $y$, if $x$ is ranked ahead of $y$ by attribute $a$, and at the same time, $x$ is not ranked ahead of $y$ by attribute $b$, then $x$ is ranked ahead of $y$ by attribute $c$.

In order to facilitate readability we introduce three new notations $p, q, r$, where:

$$
\begin{aligned}
& p \text { stands for } x \succ_{\{a\}} y, \\
& q \text { stands for } x \preceq_{\{b\}} y, \text { and } \\
& r \text { stands for } x \succ_{\{c\}} y .
\end{aligned}
$$

Obviously, $p, q, r$ generate six implications where one of them implies any of the other two. When two of them imply the third we obtain a rule similar to

$$
x \succ_{\{a\}} y \wedge x \preceq_{\{b\}} y \Rightarrow x \succ_{\{c\}} y .
$$

The three possible rules are illustrated in Fig. 1, Fig. 2, and Fig. 3. They can be used to draw conclusions when only 


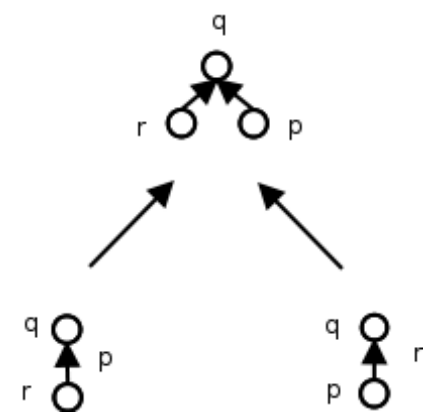

Fig. 2. $\quad p$ and $r$ imply $q$

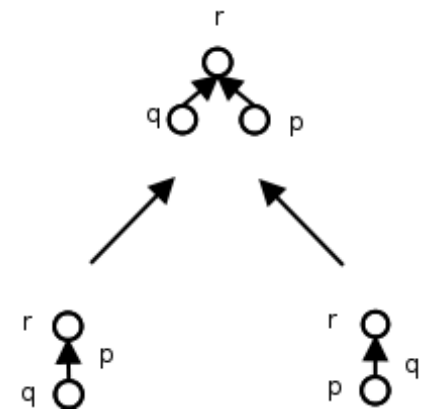

Fig. 3. $\quad p$ and $q$ imply $r$

couples of spaces are evaluated and also when a conclusion involving all of them is required.

We can extract much more information if we connect all these implications as in Fig. 4. The applied implication rules are based on the theory presented in Section II.

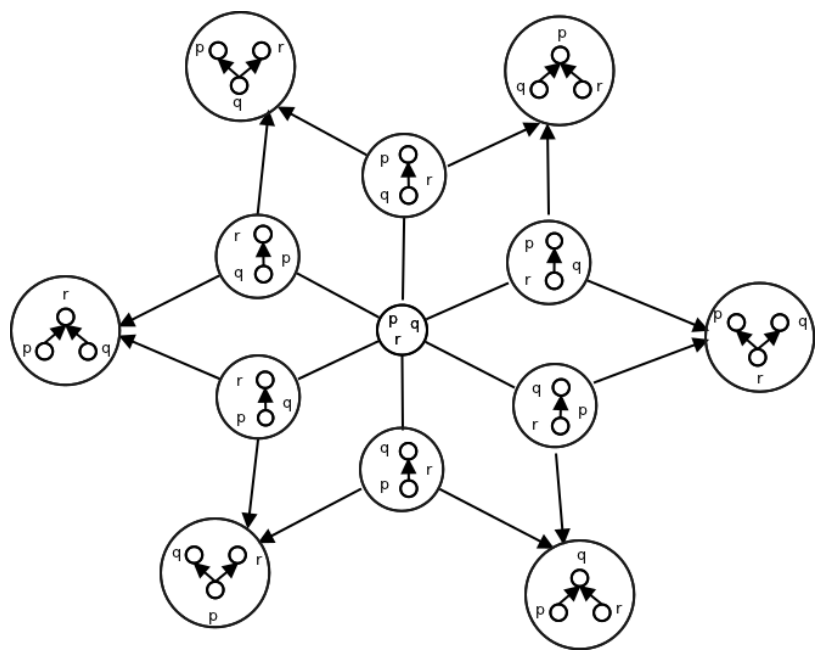

Fig. 4. Illustration of ordering rules

The graph in Fig. 5 shows that comparing two cases where an element is a rule's antecedent in one case or a rule's consequent in the other one is operating with two disjoint sets of implications.

Any two non disjoint sets of implications, i.e. pares of implications sharing one implication, generate a rule where

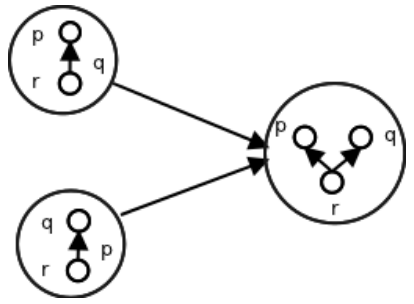

Fig. 5. $\quad p$ and $q$ are implied by $r$

one element is an antecedent and another rule where another element is a consequent, see f. ex. Fig. 6.

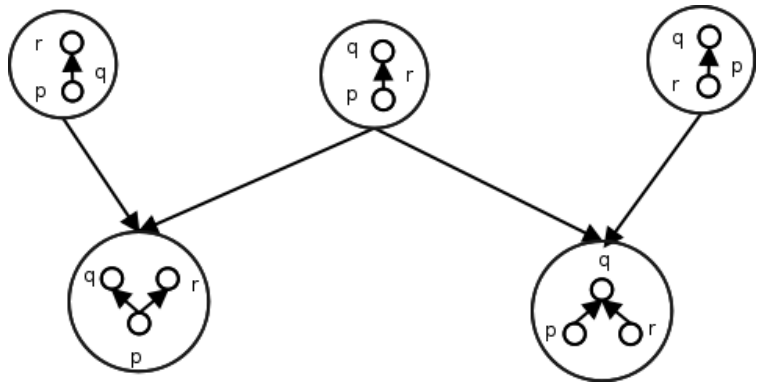

Fig. 6. Two sets of implications

Formal classroom space shapes the behavior of lecturers and students, [3]. A ranking system can be used for drawing automated conclusions when different initial rules are assumed. For ranking systems the rule illustrated in Fig. 6 can be used to show how one ordering combined with two different orderings can lead to two different conclusions.

Remark: Learning spaces should not be considered as a part of knowledge space theory, [6] and [8]. Knowledge spaces refer to states of knowledge of a person.

\section{CONCLUSION}

Appropriate combination of ordering rules sharing common elements leads to new conclusions. Closed sets coupled with ordering rules can be applied in decision support processes and development of machine learning techniques.

In future work we intend to investigate opportunities to involve more than three elements and rules in an ordering rules deductive system.

\section{REFERENCES}

[1] K. J. Arrow, Social Choice and Individual Values, Wiley, New York, 2nd ed. 1963.

[2] K. P. Bogart, Some social sciences applications of ordered sets, In: I. Rival, Editor, Ordered Sets, Reidel, Dordrecht, pp. 759-787, 1982.

[3] D. C. Brooks, Space and Consequences: The Impact of Different Formal Learning Spaces on Instructor and Student Behaviour, Journal of Learning Spaces, Vol.1, No 2, 2012.

[4] C. Carpineto and G. Romano, Concept Data Analysis: Theory and Applications, John Wiley and Sons, Ltd., 2004.

[5] B. A. Davey and H. A. Priestley, Introduction to lattices and order, Cambridge University Press, Cambridge, 2005.

[6] J.-P. Doignon and J.-C. Falmagne, Knowledge Spaces, Springer-Verlag, 1999.

49 | P a g e 
[7] J.-P. Doignon and J.-C. Falmagne, Learning Spaces, Springer-Verlag, 2011.

[8] J. -C. Falmagne, D. Albert, C. Doble, D. Eppstein, and X. Hu, Knowledge Spaces: Applications in Education, Springer, 2013.

[9] Z. Pawlak, Rough Sets, Theoretical Aspects of Reasoning about Data, Kluwer Academic Publishers, Dordrecht, 1991.

[10] Y. Sai and Y. Y. Yao, Analyzing and Mining Ordered Information Tables, Journal of Computer Science and Technology, vol. 18, No.6, 771-779, 2003.

[11] Y. Sai and Y. Y. Yao, On Mining Ordering Rules, New Frontiers in Artificial Intelligence, Lecture Notes in Computer Science, vol. 2253, pp. 316-321, 2001.

[12] K. Yordzhev and I. Peneva, Computer Administering of the Psychological Investigations: Set-Relational Representation, Open Journal of Applied Sciences, 2, pp. 110-114, 2012. http://www.scirp.org/journal/PaperInformation.aspx?PaperID=20331 